\title{
Existence of positive solutions to a non-positive elastic beam equation with both ends fixed
}

\author{
Haixia Lu', Li Sun ${ }^{2,3^{*}}$ and Jingxian Sun ${ }^{2}$
}

* Correspondence: slwgw7653@xznu.edu.cn

${ }^{2}$ Department of Mathematics, Jiangsu Normal University, Xuzhou, Jiangsu 221116, P. R. China

Full list of author information is available at the end of the article

\begin{abstract}
This article is concerned with the existence of nontrivial solutions for a non-positive fourth-order two-point boundary value problem (BVP) and the existence of positive solutions for a semipositive fourth-order two-point BVP. In mechanics, the problem describes the deflection of an elastic beam rigidly fixed at both ends. The method to show our main results is the topological degree and fixed point theory of nonlinear operator on lattice.

Mathematics Subject Classification 2010: 34B18; 34B16; $34 B 15$.
\end{abstract}

Keywords: lattice, topological degree, fixed point, nontrivial solutions and positive solutions, elastic beam equations

\section{Introduction}

The purpose of this article is to investigate the existence of nontrivial solutions and positive solutions of the following nonlinear fourth-order two-point boundary value problem (for short, BVP)

$$
\left\{\begin{array}{c}
u^{(4)}(t)=\lambda f(t, u(t)), \quad 0 \leq t \leq 1, \\
u(0)=u(1)=u^{\prime}(0)=u^{\prime}(1)=0
\end{array}\right.
$$

where $\lambda$ is a positive parameter, $f:[0,1] \times R^{1} \rightarrow R^{1}$ is continuous.

Fourth-order two-point BVPs are useful for material mechanics because the problems usually characterize the deflection of an elastic beam. The problem $(P)$ describes the deflection of an elastic beam with both ends rigidly fixed. The existence and multiplicity of positive solutions for the elastic beam equations has been studied extensively when the non-linear term $f:[0,1] \times[0,+\infty) \rightarrow[0,+\infty)$ is continuous, see for example [1-10] and references therein. Agarwal and Chow [1] investigated problem $(P)$ by using of contraction mapping and iterative methods. Bai [3] applied upper and lower solution method and Yao [9] used Guo-Krasnosel'skii fixed point theorem of cone expansioncompression type. However, there are only a few articles concerned with the nonpositive or semipositive elastic beam equations. Yao [11] considered the existence of positive solutions of semipositive elastic beam equations by constructing control functions and a special cone and using fixed point theorem of cone expansion-compression type. In this article, we assume that $f:[0,1] \times R^{1} \rightarrow R^{1}$, which implies the problem $(P)$ is nonpositive (or semipositive particularly). By the topological degree and fixed point theory of superlinear operator on lattice (the definition of lattice will be given in

(c) 2012 Lu et al; licensee Springer. This is an Open Access article distributed under the terms of the Creative Commons Attribution License (http://creativecommons.org/licenses/by/2.0), which permits unrestricted use, distribution, and reproduction in any medium, provided the original work is properly cited. 
Section 2), we obtain the existence of nontrivial solutions for the non-positive BVP $(P)$ and the existence of positive solutions for the semipositive BVP $(P)$.

\section{Preliminaries}

Let $E$ be an ordered Banach space in which the partial ordering $\leq$ is induced by a cone $P \subset E, \theta$ denote the zero element of $E$. $P$ is called solid if int $P \neq \varnothing$, i.e., $P$ has nonempty interior. $P$ is called a generating cone if $E=P-P$. For the concepts and properties about the cones we refer to [12-14].

We call $E$ a lattice in the partial ordering $\leq$, if for arbitrary $x, y \in E$, $\sup \{x, y\}$ and $\inf$ $\{x, y\}$ exist. For $x \in E$, let

$$
x^{+}=\sup \{x, \theta\}, x^{-}=\sup \{-x, \theta\},
$$

which are called the positive and the negative part of $x$, respectively. Take $|x|=x^{+}+$ $x^{-}$, then $|x| \in P$, and $|x|$ is called the module of $x$. One can see [15] for the definition and the properties about the lattice.

For convenience, we use the following notations:

$$
x_{+}=x^{+}, x_{-}=-x^{-},
$$

then

$$
x_{+} \in P, x_{-} \in(-P), x=x_{+}+x_{-} .
$$

Remark 2.1 If $E$ is a lattice, then $P$ is a generating cone.

Definition 2.1 [[16], Definition 3.2, p. 929]. Let $D \subset E$ and $F: D \rightarrow E$ be a nonlinear operator. $F$ is said to be quasi-additive on lattice, if there exists $y_{0} \in E$ such that

$$
F x=F x_{+}+F x_{-}+y_{0}, \quad \forall x \in D,
$$

where $x_{+}$and $x_{-}$are defined by (2.1).

Remark 2.2 We point out that the condition (2.2) appears naturally in the applications of nonlinear differential equations and integral equations.

Let

$$
E=C[a, b]=\left\{x(t) \mid x:[a, b] \rightarrow R^{1} \text { is continuous }\right\},
$$

and $f:[a, b] \times R^{1} \rightarrow R^{1}$. Consider the Nemytskii operator

$$
(F x)(t)=f(t, x(t)) .
$$

Set $P=\{x \in C[a, b] \mid x(t) \geq 0\}$, then $E=C[a, b]$ is a lattice in the partial ordering which is induced by $P$. For any $x \in C[a, b]$, it is evident that

$$
x_{+}(t)=\max \{x(t), 0\}, x_{-}(t)=\min \{x(t), 0\},
$$

and hence $|x|(t)=|x(t)|$. By Remark 3.1 in [16], we know that there exists $y_{0} \in C[a$, b] such that $F x=F x_{+}+F x_{-}+y_{0}, \forall x \in C[a, b]$.

Suppose that $B$ is a linear operator and $A=B F$. It follows that

$$
A x=A x_{+}+A x_{-}+B y_{0}
$$

which means that $A$ is quasi-additive on lattice. 
Definition 2.2 [[17], Definition, p. 261]. Let $B: E \rightarrow E$ be a linear operator. $B$ is said to be a $u_{0}$-bounded linear operator, if there exists $u_{0} \in P \backslash\{\theta\}$, such that for any $x \in P$ $\backslash\{\theta\}$, there exist a natural number $n$ and real numbers $\zeta, \eta>0$, such that

$$
\zeta u_{0} \leq B^{n} x \leq \eta u_{0} .
$$

Lemma 2.1 [[18], Theorem 4.2.2, p. 122]. Let $P$ be a generating cone and $B$ a $u_{0^{-}}$ bounded completely continuous linear operator. Then the spectral radius $r(B) \neq 0$ and $r^{-1}(B)$ is the only positive eigenvalue corresponding to positive eigenvectors and $B$ has no other eigenvectors except those corresponding to $r^{-1}(B)$.

Let $B: E \rightarrow E$ be a positive completely continuous linear operator, $r(B)$ a spectral radius of $B, B^{*}$ the conjugated operator of $B$, and $P^{*}$ the conjugated cone of $P$. Since $P$ $\subset E$ is a generating cone, according to the famous Krein-Rutman theorem (see [14]), if $r(B) \neq 0$, then there exist $\bar{\varphi} \in P \backslash\{\theta\}$, and $g^{* *} \in P^{*} \backslash\{\theta\}$, such that

$$
B \bar{\varphi}=r(B) \bar{\varphi}, \quad B^{*} g^{*}=r(B) g^{*} .
$$

Fix $\bar{\varphi} \in P \backslash\{\theta\}, g^{*} \in P^{*} \backslash\{\theta\}$ such that (2.3) holds. For $\delta>0$, let

$$
P\left(g^{*}, \delta\right)=\left\{x \in P, g^{*}(x) \geq \delta\|x\|\right\},
$$

then $P\left(g^{*}, \delta\right)$ is also a cone in $E$.

Definition 2.3 [[19], Definition, p. 528]. Let $B$ be a positive linear operator. $B$ is said to satisfy $H$ condition, if there exist $\bar{\varphi} \in P \backslash\{\theta\}, g^{* *} \in P^{*} \mid\{\theta\}$ and $\delta>0$ such that (2.3) holds, and $B$ maps $P$ into $P\left(g^{*}, \delta\right)$.

Remark 2.3 Let $B \varphi=\int_{a}^{b} k(x, y) \varphi(y) d y$, where $k(x, y) \in C([a, b] \times[a, b]), k(x, y) \geq 0$, $\phi \in C[a, b]$. Suppose that (2.3) holds and there exists $v(x) \in P \backslash\{\theta\}$ such that

$$
k(x, y) \geq v(x) k(\tau, y), \quad \forall x, y, \tau \in[a, b]
$$

and $v(x) g^{*}(x) \otimes 0$, then $B$ satisfies $H$ condition (see [19]).

Lemma 2.2 [[16], Theorem 3.1, p. 929]. Let $P$ be a solid cone, $A: E \rightarrow E$ be a completely continuous operator satisfying $A=B F$, where $F$ is quasi-additive on lattice, $B$ is a positive bounded operator satisfying $H$ condition. Suppose that

(i) there exist $a_{1}>r^{-1}(B), y_{1} \in P$ such that

$$
F x \geq a_{1} x-y_{1}, \quad \forall x \in P
$$

(ii) there exist $0 \leq a_{2} \leq r^{-1}(B), y_{2} \in P$ such that

$$
F x \geq a_{2} x-y_{2}, \quad \forall x \in(-P) .
$$

Then there exists $R_{0}>0$ such that $\operatorname{deg}\left(I-A, T_{R}, \theta\right)=0$ for any $R>R_{0}$, where $T_{R}=$ $\{x \in C[0,1]:\|x\|<R\}$.

Lemma 2.3 [[16], Theorem 3.3, p. 932]. Let $\Omega$ be a bounded open subset of $E$, $\theta \in$ $\Omega$, and $A: \bar{\Omega} \rightarrow P$ a completely continuous operator. Suppose that $A$ has no fixed point on $\partial \Omega$. If

(i) there exists a positive bounded linear operator $B$ such that $|A x| \leq B|x|$, for all $x \in$ $\partial \Omega$;

(ii) $r(B) \leq 1$.

Then $\operatorname{deg}(I-A, \Omega, \theta)=1$. 


\section{Existence of nontrivial solutions for the non-positive BVP $(P)$}

In the sequel we always take $E=C[0,1]$ with the norm $\|u\|=\max _{0 \leq t \leq 1}|u(t)|$ and $P=$ $\{u \in C[0,1] \mid u(t) \geq 0,0 \leq t \leq 1\}$. Then $P$ is a solid cone in $E$ and $E$ is a lattice under the partial ordering $\leq$ induced by $P$.

A solution of BVP $(P)$ is a fourth differentiable function $u:[0,1] \rightarrow R$ such that $u$ satisfies $(P) . u$ is said to be a positive solution of BVP $(P)$ if $u(t)>0,0<t<1$. Let $G(t$, $s$ ) be Green's function of homogeneous linear problem $u^{(4)}(t)=0, u(0)=u(1)=u^{\prime}(0)=$ $u^{\prime}(1)=0$. From Yao [11] we have

$$
G(t, s)=\left\{\begin{array}{l}
\frac{1}{6} t^{2}(1-s)^{2}[(s-t)+2(1-t) s], 0 \leq t \leq s \leq 1 \\
\frac{1}{6} s^{2}(1-t)^{2}[(t-s)+2(1-s) t], 0 \leq s \leq t \leq 1,
\end{array}\right.
$$

and

$\left(\mathrm{G}_{1}\right) G(t, s) \geq 0,0 \leq t, s \leq 1$;

$\left(\mathrm{G}_{2}\right) G(t, s)=G(s, t)$;

$\left(\mathrm{G}_{3}\right) G(t, s) \geq p(t) G(\tau ; s), 0 \leq t, s, \tau \leq 1$, where $p(t)=\frac{2}{3} \min \left\{t^{2},(1-t)^{2}\right\}$.

Lemma 3.1 Let $H(t)=\frac{1}{2} t^{2}(1-t)^{2}$ and $q(s)=\frac{2}{3} s^{2}(1-s)^{2}$. Then

$$
q(s) H(t) \leq G(t, s) \leq H(t), \quad 0 \leq t, s \leq 1 .
$$

Proof. Since $G(0, s)=G(1, s)=0,0 \leq s \leq 1, H(0)=H(1)=0$, then $q(s) H(t)=G(t, s)$ $=H(t)$ holds for $t=0$ and $t=1$. If $0<t \leq s \leq 1$ and $t<1$, then

$$
\begin{aligned}
G(t, s)= & \frac{1}{6} t^{2}(1-s)^{2}[3 s(1-t)-t(1-s)] \\
& \leq \frac{1}{2} t^{2}(1-s)^{2} s(1-t) \\
& \leq \frac{1}{2} t^{2}(1-t)^{2} s(1-s) \\
& <\frac{1}{2} t^{2}(1-t)^{2}=H(t)
\end{aligned}
$$

and

$$
\begin{aligned}
\frac{G(t, s)}{H(t)} & =\frac{\frac{1}{6} t^{2}(1-s)^{2}[3 s(1-t)-t(1-s)]}{\frac{1}{2} t^{2}(1-t)^{2}} \\
& \geq \frac{(1-s)^{2}[3 s(1-t)-t(1-t)]}{3(1-t)^{2}} \\
& =\frac{(1-s)^{2}(3 s-t)}{3(1-t)} \geq \frac{2 s(1-s)^{2}}{3(1-t)} \\
& \geq \frac{2}{3} s^{2}(1-s)^{2}=q(s) .
\end{aligned}
$$

Similarly, (3.1) holds for $0 \leq s \leq t<1$ and $t>0$. The proof is complete. $\quad \square$ It is well known that the problem $(P)$ is equivalent to the integral equation

$$
u(t)=\lambda \int_{0}^{1} G(t, s) f(s, u(s)) d s .
$$

Let

$$
(A u)(t)=\int_{0}^{1} G(t, s) f(s, u(s)) d s
$$




$$
(B u)(t)=\int_{0}^{1} G(t, s) u(s) d s .
$$

Lemma 3.2 Let $B$ be defined by (3.3). Then $B$ is a $u_{0}$-bounded linear operator.

Proof. Let $u_{0}(t)=H(t)=\frac{1}{2} t^{2}(1-t)^{2}, t \in[0,1]$. For any $u \in P \backslash\{\theta\}$, by Lemma 3.1 we have

$$
\int_{0}^{1} q(s) H(t) u(s) d s \leq B u(t)=\int_{0}^{1} G(t, s) u(s) d s \leq \int_{0}^{1} H(t) u(s) d s .
$$

Take arbitrarily $0<\varepsilon_{0}<\frac{1}{2}$, then

$$
u_{0}(t) \int_{\varepsilon_{0}}^{1-\varepsilon_{0}} q(s) u(s) d s \leq B u(t) \leq\|u\| \cdot u_{0}(t) .
$$

Let $\zeta=\int_{\varepsilon_{0}}^{1-\varepsilon_{0}} q(s) u(s) d s>0, \eta=\|u\|>0$. Then

$$
\zeta u_{0} \leq B u \leq \eta u_{0}
$$

This indicates that $B: E \rightarrow E$ is a $u_{0}$-bounded linear operator. $\square$

From Lemma 2.1 we have $r(B) \neq 0$ and $r^{-1}(B)$ is the only eigenvalue of $B$. Denote $\lambda_{1}$ $=r^{-1}(B)$.

Now let us list the following conditions which will be used in this article:

$\left(\mathrm{H}_{1}\right)$ there exist constants $\alpha$ and $\beta$ with $\alpha>\beta \geq 0$ satisfying

$$
\liminf _{u \rightarrow+\infty} \frac{f(t, u)}{u} \geq \alpha, \limsup _{u \rightarrow-\infty} \frac{f(t, u)}{u} \leq \beta, \quad \forall t \in[0,1] .
$$

$\left(\mathrm{H}_{2}\right)$ there exists a constant $\gamma \geq 0$ satisfying

$$
\underset{u \rightarrow 0}{\limsup }\left|\frac{f(t, u)}{u}\right| \leq \gamma, \quad \forall t \in[0,1]
$$

$\left(\mathrm{H}_{3}\right) \lim _{u \rightarrow+\infty} \frac{f(t, u)}{u}=+\infty$.

Theorem 3.1 Suppose that $\left(\mathrm{H}_{1}\right)$ and $\left(\mathrm{H}_{2}\right)$ hold. Then for any $\lambda \in\left(\frac{\lambda_{1}}{\alpha}, \frac{\lambda_{1}}{l}\right)$, BVP $(P)$ has at least one nontrivial solution, where $\lambda_{1}=r^{-1}(B)$ is the only eigenvalue of $B, B$ is denoted by (3.3), $\iota=\max \{\beta, \gamma\}$.

Proof. Let $(F u)(t)=f(t, u(t))$. Then $A=B F$, where $A$ is denoted by (3.2). By Remark 2.2, $F$ is quasi-additive on lattice. Applying the Arzela-Ascoli theorem and a standard argument, we can prove that $A: E \rightarrow E$ is a completely continuous operator.

Now we show that $\lambda A=\lambda B F$ has at least one nontrivial fixed point, which is the nontrivial solution of BVP $(P)$.

On account of $\left(\mathrm{G}_{3}\right)$ we have that $p(t)=\frac{2}{3} \min \left\{t^{2},(1-t)^{2}\right\} \in P \backslash\{\theta\}$ such that

$$
G(t, s) \geq p(t) G(\tau, s), \quad 0 \leq t, s, \tau \leq 1 .
$$


Notice that $B u(t)=\int_{0}^{1} G(t, s) u(s) d s$, where $G(t, s) \geq 0, G(t, s) \in C([0,1] \times[0,1])$. From Lemma $3.2 B$ is a $u_{0}$-bounded linear operator. By Lemma 2.1 we have $r(B) \neq 0$ and $\lambda_{1}=r^{-1}(B)$ is the only eigenvalue of $B$. Then there exist $\bar{\varphi} \in P \backslash\{\theta\}$ and $g^{*} \in P^{*} \backslash\{\theta\}$ such that (2.3) holds. Notice that $\lambda>0$, from Remark 2.3, $\lambda B$ satisfies $H$ condition.

By (3.4) and (3.5), there exist $r>0, M>0$ and $0<\varepsilon<\min \left\{\frac{\alpha \lambda-\lambda_{1}}{\lambda}, \frac{\lambda_{1}-\beta \lambda}{\lambda}, \frac{\lambda_{1}-\gamma \lambda}{\lambda}\right\}$ such that

$$
\begin{array}{ll}
f(t, u) \geq(\alpha-\varepsilon) u-M, \quad \forall t \in[0,1], & u \geq 0, \\
f(t, u) \geq(\beta+\varepsilon) u-M, \quad \forall t \in[0,1], & u \leq 0, \\
|f(t, u)| \leq(\gamma+\varepsilon)|u|, \quad \forall t \in[0,1], & |u| \leq r .
\end{array}
$$

By (3.6) and (3.7), we have (2.4) and (2.5) hold, where $a_{1}=\alpha-\varepsilon, a_{2}=\beta+\varepsilon$.

Let $B_{1}=\lambda B$. Then $r^{-1}\left(B_{1}\right)=\frac{\lambda_{1}}{\lambda}$. Obviously, for any $\lambda \in\left(\frac{\lambda_{1}}{\alpha}, \frac{\lambda_{1}}{l}\right), a_{1}>r^{-1}\left(B_{1}\right), a_{2}<r$ ${ }^{1}\left(B_{1}\right)$. From Lemma 2.2 there exists $R_{0}>0$ such that for any $R>\max \left\{R_{0}, r\right\}$,

$$
\operatorname{deg}\left(I-\lambda A, T_{R}, \theta\right)=0 .
$$

Let $B_{2}=\lambda(\gamma+\varepsilon) B$. From (3.8) we have $|\lambda A u| \leq B_{2}|u|$, also $r\left(B_{2}\right)=\frac{\lambda(\gamma+\varepsilon)}{\lambda_{1}} \leq 1$. Without loss of generality we assume that $\lambda A$ has no fixed point on $\partial T_{r}$, where $T_{r}=\{u \in C$ $[0,1] \mid\|u\|<r\}$. By Lemma 2.3 we have

$$
\operatorname{deg}\left(I-\lambda A, T_{r}, \theta\right)=1,
$$

It is easy to see from (3.9) and (3.10) that $\lambda A$ has at least one nontrivial fixed point. Thus problem $(P)$ has at least one nontrivial solution. $\square$

Remark 3.1 If $\alpha=+\infty, \beta=\gamma=0$, then for any $\lambda>0$ problem $(P)$ has at least one nontrivial solution.

Theorem 3.2 Suppose that $\left(\mathrm{H}_{1}\right)$ holds. Assume $f(t, 0) \equiv 0, \forall t \in[0,1]$ and

$$
\lim _{u \rightarrow 0} \frac{f(t, u)}{u}=\rho
$$

Then for any $\lambda \in\left(\frac{\lambda_{1}}{\alpha}, \frac{\lambda_{1}}{\beta}\right)$ and $\lambda \neq \frac{\lambda_{1}}{\rho}$, BVP $(P)$ has at least one nontrivial solution.

Proof. Since $f(t, 0) \equiv 0, \forall t \in[0,1]$, then $A \theta=\theta$. By (3.11) we have that the Frechet derivative $A_{\theta}^{\prime}$ of $A$ at $\theta$ exists and

$$
\left(A_{\theta}^{\prime} u\right)(t)=\int_{0}^{1} G(t, s) \rho u(s) d s .
$$

Notice that $\lambda \neq \frac{\lambda_{1}}{\rho}$, then 1 is not an eigenvalue of $\lambda A_{\theta}^{\prime}$. By the famous Leray-Schauder theorem there exists $r>0$ such that

$$
\operatorname{deg}\left(I-\lambda A, T_{r}, \theta\right)=\operatorname{deg}\left(I-\lambda A_{\theta}^{\prime}, T_{r}, \theta\right)=(-1)^{\kappa} \neq 0,
$$

where $\kappa$ is the sum of algebraic multiplicities for all eigenvalues of $\lambda A_{\theta}^{\prime}$ lying in the interval $(0,1)$. From the proof of Theorem 3.1 we have that (3.9) holds for any 
$\lambda \in\left(\frac{\lambda_{1}}{\alpha}, \frac{\lambda_{1}}{\beta}\right)$. By (3.9) and (3.12), $\lambda A$ has at least one nontrivial fixed point. Thus problem $(P)$ has at least one nontrivial solution. $\square$

\section{Existence of positive solutions for the semipositive BVP (P)}

In many real problems, the positive solution is more significant. In this section we will study this kind of question.

Lemma 4.1 [[20], Theorem 1, p. 90]. Let $D=[a, b]$. Suppose

(i) $G(t, s)$ is a symmetric kernel. And there exist $D_{0} \subset D$, mes $D_{0} \neq 0$ and $\delta>0$ such that

$$
G(t, s) \geq \delta G(\tau, s), \quad \forall t \in D_{0}, s, \tau \in D ;
$$

(ii) $f(t, u)$ is bounded from below and $\lim _{u \rightarrow+\infty} f(t, u)=+\infty$ uniformly holds for $t \in D_{0}$. Then for any $\lambda^{*}>0$, there exists $R=R\left(\lambda^{*}\right)>0$ such that if $0<\lambda_{0} \leq \lambda^{*},\left\|\phi_{0}\right\| \geq R$ and $\phi_{0}=\lambda_{0} A \phi_{0}$, then $\phi_{0}(x) \geq 0$, where $A$ is denoted by (3.2).

Theorem 4.1 Suppose that $\left(\mathrm{H}_{3}\right)$ holds. Then there exists $\lambda^{*}>0$ such that for any 0 $<\lambda<\lambda^{*}$ BVP $(P)$ has at least one positive solution.

Proof. By $\left(\mathrm{H}_{3}\right)$ there exists a constant $b>0$ such that

$$
f(t, u) \geq-b, \quad \forall t \in[0,1], \quad u \geq 0 .
$$

Let

$$
f_{1}(t, u)=\left\{\begin{array}{rr}
f(t, u), \quad u \geq 0 \\
f(t,-u), \quad u<0
\end{array}\right.
$$

From (4.1) $f_{1}$ is bounded from below. Let

$$
\left(A_{1} u\right)(t)=\int_{0}^{1} G(t, s) f_{1}(s, u(s)) d s .
$$

Then $A_{1}: E \rightarrow E$ is a completely continuous operator.

From the proof of Theorems 2.7.3 and 2.7.4 in Sun [18], there exists $R_{0}>0$ such that for any $R>R_{0}$,

$$
\operatorname{deg}\left(I-\lambda A_{1}, T_{R}, \theta\right)=0, \quad \forall \lambda>0 .
$$

Take $0<r<R_{0}$. Let $m=\max _{0 \leq t \leq 1,|u|<r}\left|f_{1}(t, u)\right|, G=\max _{0 \leq s, t \leq 1} G(t, s), \bar{\lambda}=r(m G)^{-1}$. For any $0<\lambda<\bar{\lambda}, u \in \partial T_{r}$, we have

$$
\begin{aligned}
\left\|\lambda A_{1} u\right\| & =\max _{0 \leq t \leq 1}\left|\int_{0}^{1} \lambda G(t, s) f_{1}(s, u(s)) d s\right| \\
& <\bar{\lambda} G m=r=\|u\| .
\end{aligned}
$$

Thus

$$
\operatorname{deg}\left(I-\lambda A_{1}, T_{r}, \theta\right)=1, \quad \forall 0<\lambda<\bar{\lambda} .
$$

From (4.2) and (4.3), we have that for any $0<\lambda<\bar{\lambda}$, there exist $u_{\lambda,} \in C[0,1],\left\|u_{\lambda}\right\|$ $>r$ such that $u_{\lambda}=\lambda A_{1} u_{\lambda}$. In order to apply Lemma 4.1 we claim that 


$$
\lim _{\lambda \rightarrow 0^{+}, u_{\lambda}=\lambda A_{1} u_{\lambda},\left\|u_{\lambda}\right\|>r}\left\|u_{\lambda}\right\|=+\infty
$$

In fact, if (4.4) doesn't hold, then there exist $\lambda_{n}>0, u_{\lambda_{n}} \in C[0,1]$ such that $\lambda_{n} \rightarrow 0$, $r<\left\|u_{\lambda_{n}}\right\|<c(c>0$ is a constant $)$ and

$$
u_{\lambda_{n}}=\lambda_{n} A_{1} u_{\lambda_{n}}
$$

Since $A_{1}$ is completely continuous, then $\left\{u_{\lambda_{n}}\right\}$ has a subsequence converging to $u^{*} \in$ $C[0,1]$. Assume, without loss of generality, that it is $\left\{u_{\lambda_{n}}\right\}$. Taking $n \rightarrow+\infty$ in (4.5), we have $u^{*}=\theta$, which is a contradiction to $\left\|u_{\lambda_{n}}\right\|>r>0$. Hence (4.4) holds.

Let $D=[0,1], D_{0}=\left[t_{1}, t_{2}\right] \subset(0,1) \subset D, \delta=\min _{t_{1} \leq t \leq t_{2}} p(t)>0$. By $\left(\mathrm{G}_{3}\right)$

$$
G(t, s) \geq \delta G(\tau, s), \quad \forall t \in D_{0}, s, \tau \in D
$$

From $\left(\mathrm{H}_{3}\right)$ and the definition of $f_{1}$, we have

$$
\lim _{u \rightarrow+\infty} f_{1}(t, u)=\lim _{u \rightarrow+\infty} f(t, u)=+\infty
$$

By Lemma 4.1 there exists $R=R(\bar{\lambda})>0$ such that if $0<\lambda \leq \bar{\lambda},\left\|u_{\lambda}\right\| \geq R$ and $u_{\lambda}=$ $\lambda A_{1} u_{\lambda}$, then $u_{\lambda}(t) \geq 0$. By (4.4), there exists $\lambda^{*}<\bar{\lambda}$ such that if $0<\lambda \leq \lambda^{*},\left\|u_{\lambda}\right\| \geq r$ and $u_{\lambda}=\lambda A_{1} u_{\lambda}$, then $\left\|u_{\lambda}\right\| \geq R$. Thus $u_{\lambda}(t) \geq 0$. By the definitions of $A_{1}$ and $f_{1}$ we have

$$
u_{\lambda}(t)=\lambda A_{1} u_{\lambda}(t)=\lambda \int_{0}^{1} G(t, s) f_{1}(s, u(s)) d s=\lambda \int_{0}^{1} G(t, s) f(s, u(s)) d s=\lambda A u_{\lambda}(t)
$$

And so $u_{\lambda}(t)$ is a positive solution of problem $(P)$. $\square$

Remark 4.1 In Theorem 4.1, without assuming that $f(t, u) \geq 0$ when $u \geq 0$, we obtain the existence of positive solutions for the semipositive BVP $(P)$.

Remark 4.2 Noticing that, in this article, we only study the existence of positive solutions for BVP $(P)$, which is irrelevant to the value of $f(t, u)$ when $u \leq 0$, we only need to suppose that $f(t, u)$ is bounded from below when $u \geq 0$. In fact, $f(t, u)$ may be unbounded from below when $u \leq 0$.

\section{Two examples}

In order to illustrate possible applications of Theorems 3.2 and 4.1, we give two examples.

Example 5.1 Consider the fourth-order BVP

$$
\left\{\begin{array}{l}
u^{(4)}(t)=\lambda[\sin u(t)+u(t) \arctan u(t)+\pi u(t)], \quad 0 \leq t \leq 1 \\
u(0)=u(1)=u^{\prime}(0)=u^{\prime}(1)=0
\end{array}\right.
$$

In this example, $f(t, u)=\sin u+u \arctan u+\pi u$, then

$$
\begin{aligned}
& \liminf _{u \rightarrow+\infty} \frac{f(t, u)}{u}=\frac{3 \pi}{2}, \quad \liminf _{u \rightarrow-\infty} \frac{f(t, u)}{u}=\frac{\pi}{2}, \\
& \lim _{u \rightarrow 0} \frac{f(t, u)}{u}=1+\pi .
\end{aligned}
$$


Take $\alpha=3 \pi / 2, \beta=\pi / 2, \rho=\pi+1$. Then (5.1), (5.2) indicate $\left(\mathrm{H}_{1}\right)$, (3.11) hold, repectively. Notice that $\alpha>\rho>\beta>0$ and $f(t, 0) \equiv 0, \forall t \in[0,1]$, by Theorem 3.2 for any $\lambda \in\left(\frac{\lambda_{1}}{\alpha}, \frac{\lambda_{1}}{\beta}\right)$ and $\lambda \neq \frac{\lambda_{1}}{\rho}$, BVP $\left(P_{1}\right)$ has at least one nontrivial solution.

Example 5.2 Consider the fourth-order BVP

$$
\left\{\begin{array}{l}
u^{(4)}(t)=\lambda\left[(\sqrt{t}+1) u^{2}-\sqrt[3]{u}\right], \quad 0 \leq t \leq 1, \\
u(0)=u(1)=u^{\prime}(0)=u^{\prime}(1)=0 .
\end{array}\right.
$$

In this example, $f(t, u)=(\sqrt{t}+1) u^{2}-\sqrt[3]{u}$, then

$$
\lim _{u \rightarrow+\infty} \frac{f(t, u)}{u}=\lim _{u \rightarrow+\infty}\left[(\sqrt{t}+1) u-\frac{1}{\sqrt[3]{u^{2}}}\right]=+\infty .
$$

(5.3) means $\left(H_{3}\right)$ holds. By Theorem 4.1 there exists $\lambda^{*}>0$ such that for any $0<\lambda<$ $\lambda * \operatorname{BVP}\left(P_{2}\right)$ has at least one positive solution.

\section{Acknowledgements}

The authors would like to thank the referees for carefully reading this article and making valuable comments and suggestions. This work is supported by the Foundation items: NSFC $(10971179,11026203)$, NSF (BK2011202) of the Jiangsu Province, NSF (09XLR04) of the Xuzhou Normal University and NSF (2010KY07) of the Suqian College.

\section{Author details}

${ }^{1}$ Department of Teachers Education, Suqian College, Suqian, Jiangsu 223800, P. R. China ${ }^{2}$ Department of Mathematics, Jiangsu Normal University, Xuzhou, Jiangsu 221116, P. R. China ${ }^{3}$ School of Mechanics and Civil Engineering, China University of Mining \& Technology, Xuzhou, Jiangsu 221008, P. R. China

\section{Authors' contributions}

All authors typed, read and approved the final manuscript.

\section{Competing interests}

The authors declare that they have no competing interests.

Received: 30 November 2011 Accepted: 14 May 2012 Published: 14 May 2012

\section{References}

1. Agarwal, RP, Chow, YM: Iterative method for fourth order boundary value problem. J Comput App Math. 10, 203-217 (1984). doi:10.1016/0377-0427(84)90058-X

2. Bai, Z, Wang, H: On positive solutions of some nonlinear four-order beam equations. J Math Anal Appl. 270, 357-368 (2002). doi:10.1016/50022-247X(02)00071-9

3. Bai, Z: The upper and lower solution method for some fourth-order boundary value problems. Nonlinear Anal. 67, 1704-1709 (2007). doi:10.1016/j.na.2006.08.009

4. Dalmasso, R: Uniqueness of positive solutions for some nonlinear four-order operators. J Math Anal Appl. 201, 152-168 (1996). doi:10.1006/jmaa.1996.0247

5. Gupta, CP: Existence and uniqueness results for the bending of an elastic beam equation. Appl Anal. 26, 289-304 (1988). doi:10.1080/00036818808839715

6. Graef, R, Yang, B: Positive solutions of a nonlinear fourth order boundary value problem. Commun Appl Nonl Anal. 14, 61-73 (2007)

7. Korman, P: Uniqueness and exact multiplicity of solutions for a class of fourth-order semilinear problems. Proc R Soc Edinburg Sect A. 134, 179-190 (2004). doi:10.1017/50308210500003140

8. Lou, B: Positive solutions for nonlinear elastic beam models. Int J Math Sci. 27, 365-375 (2001). doi:10.1155/ S0161171201004203

9. Yao, Q: Existence of $\mathrm{n}$ solutions and/or positive solutions to a semipositive elastic beam equation. Nonlinear Anal. 66, 138-150 (2007). doi:10.1016/j.na.2005.11.016

10. Zhang, X: Existence and iteration of monotone positive solutions for an elastic beam equation with a corner. Nonlinear Anal. 10, 2097-2103 (2009). doi:10.1016/j.nonrwa.2008.03.017

11. Yao, Q: Positive solutions for eigenvalue problems of four-order elastic beam equations. Appl Math Lett. 17, 237-243 (2004). doi:10.1016/50893-9659(04)90037-7

12. Amann, H: Fixed point equations and nonlinear eigenvalue problems in ordered Banach spaces. SIAM Rev. 18, 620-709 (1976). doi:10.1137/1018114

13. Guo, D, Lakshmikanthan, V: Nonlinear Problems in Abstract Cones. Academic press, San Diego (1988)

14. Krein, MG, Rutman, MA: Linear operators leaving invariant a cone in a Banach space. Am Math Soc Transl. 10, 199-325 (1962)

15. Lucxemburg, WAJ, Zaanen, AC: Riesz Space, vol I. North-Holland Publishing Company, London (1971) 
16. Sun, J, Liu, X: Computation of topological degree in ordered Banach spaces with lattice structure and its application to superlinear differential equations. J Math Anal Appl. 348, 927-937 (2008). doi:10.1016/j.jmaa.2008.05.023

17. Krasnosel'skii, MA: Topological methods in the theory of nonlinear integral equations. Pergamon Press, Oxford (1964)

18. Sun, J: Nonlinear Functional Analysis and its Applications. Science Press, Beijing (2007). in Chinese

19. Sun, J: Nontrivial solutions of superlinear Hammerstein integral equations and its applications. Chinese Ann Math Ser A 7. 528-535 (1986)

20. Sun, J: Existence of positive solutions of nonlinear Hammerstein integral equations and its applications. Chinese Ann Math Ser A 9, 90-96 (1988). in Chinese

doi:10.1186/1687-2770-2012-56

Cite this article as: Lu et al: Existence of positive solutions to a non-positive elastic beam equation with both ends fixed. Boundary Value Problems 2012 2012:56.

Submit your manuscript to a SpringerOpen ${ }^{\circ}$ journal and benefit from:

- Convenient online submission

- Rigorous peer review

- Immediate publication on acceptance

- Open access: articles freely available online

- High visibility within the field

Retaining the copyright to your article

Submit your next manuscript at $\boldsymbol{\nabla}$ springeropen.com 